\title{
The culture of the body in the media space of postmodern society
}

\section{[La culture du corps dans l'espace médiatique de la société postmoderne]}

\author{
Kamil Kardis - Maria Kardis - Gabriel Pala - Tadeusz Bak - Michal Valco
}

DOI: 10.18355/XL.2021.14.04.21

\begin{abstract}
According to M. Fforde, the era of postmodernity is characterized by a form of cultural collapse, as it reveals many phenomena indicating that contemporary humans are on the path towards 'desocialization.' This process represents an overall cultural shift as well as changes in the existing anthropological paradigms. This paper is dedicated to an analysis of the issue of religion as social capital in conditions of increasing societal risks. We work with an hypothesis that sociopathological phenomena are rather determined by cultural and secularization factors.

In our article, we argue that desocialization is closely connected with current secularization processes, as well as with the progression of a materialistic view of man in compliance with the models and theories of postmodern anthropologies. We propose that the ongoing social enthropy in the form of desocialization should be dealt with holistically, i.e.,by analyzing the utilization (or the lack thereof) of religious resources for human and social flourishing.
\end{abstract}

Key words: Culture of the body, postmodernity, John Paul II, Mass Media

\section{Résumé}

Selon M. Fforde, l'ère de la postmodernité est caractérisée par une forme d'effondrement culturel, car elle révèle de nombreux phénomènes indiquant que les humains contemporains sont sur la voie de la désocialisation. Ce processus représente un changement culturel global ainsi que des changements dans la paradigmes anthropologiques existants. Cet article est consacré à une analyse de la question de la religion comme capital social dans des conditions de risques sociétaux croissants. Nous travaillons avec l'hypothèse que les phénomènes sociopathologiques sont plutôt déterminés par des facteurs culturels et de sécularisation. Dans notre article, nous soutenons que la désocialisation est étroitement liée aux processus de sécularisation actuels, ainsi qu'à la progression d'une vision matérialiste de l'homme en conformité avec les modèles et les théories des anthropologies postmodernes. Nous proposons que l'entropie sociale en cours sous forme de désocialisation soit traitée de manière holistique, c'est-à-dire en analysant l'utilisation (ou l'absence de ressources) des ressources religieuses pour l'épanouissement humain et social.

Mots clés : Culture du corps, postmodernité, Jean-Paul II, Mass Media

Introduction: Une analyse théorique du contexte culturel / moral postmoderne ${ }^{1}$ Nous vivons à une époque hautement « liquide » (Bauman, 2013), dans laquelle les règles, normes et valeurs fondamentales de la société sont progressivement transformées conformément à l'esprit des idéologies du modernisme et du

\footnotetext{
${ }^{1}$ L'article a été créé dans le cadre du projets: Progres Q05 : Aspects juridiques et sociaux de la migration et problèmes de la position des minorités à la Faculté de théologie Hussite de l'Université Charles; KEGA 040PU-4/2021 Développement du programme d'études européennes dans le deuxième cycle d'études à l'aide de méthodes didactiques innovantes ; KEGA 014PU4/2019 Support multimédia et électronique de l'éducation aux médias sous forme d'apprentissage en ligne et d'e-learning
} 
postmodernisme (Mongardini, 1992). La moralité en tant que pilier fondamental du contrôle social est en train d'être remplacée par un système juridique démocratique fondé sur un accord majoritaire. Il devient désormais évident que des valeurs fondamentales telles que la dignité humaine, la vie, le mariage, la famille, la justice, la solidarité sont radicalement remises en question et redéfinies, leur statut étant relégué à une question d'accord. Au lieu d'exercer un contrôle en limitant les possibilités, " les élites modernes liquides dominent par l'incertitude et l'insécurité », dans un environnement de « consommation insatiable, de mobilité, de contingence des relations sociales ». (Bauman - Haugaard, 2008: 111) Alors que le capital culturel précédent s'épuise, la consommation effrénée nourrie par une séduction omniprésente devient la nouvelle norme à l'horizon de l'imaginaire social.

Le contexte social de la seconde transition démographique a entraîné de profonds changements dans les orientations de valeurs et les attitudes morales de la société actuelle. Cela implique « une fertilité sous-remplacement soutenue, une multitude de modes de vie autres que le mariage, la déconnexion entre le mariage et la procréation, et pas de population stationnaire » (Lesthaeghe - Surkyn, 2008: 82), qui semblent tous être propulsés par un accent accru sur la liberté individuelle et la réalisation de soi. (Zaidi - Morgan, 2017). Dans le micro-espace d'un individu, il est devenu de plus en plus difficile de créer sa propre identité, de répondre à des questions existentielles fondamentales ou de créer un monde horizontal et vertical significatif de relations et de systèmes de soutien cohérents. Au lieu de forger l'identité d'un individu au cours $\mathrm{du}$ processus de socialisation primaire et secondaire, celle-ci est souvent déformée dans ces environnements, après avoir été influencée par la désocialisation de la famille, de l'école ou des pairs. (Giddens, 2006: 688-690) Le mésospace de la vie communautaire a également subi les conséquences de la perte de communauté (Gemeinschaft) et des processus de modernisation de la société industrielle et postindustrielle. (Bak, 2010)

Fforde (2010: 23-25) offre un exemple révélateur des manifestations concrètes de la désocialisation dans son livre Desocialisation: La crise de la postmodernité, en utilisant son étude de cas de la société britannique. Nous apprenons ici que la désocialisation implique un conflit entre les anthropologies gouvernantes. Suite à un virage énergique vers des anthropologies réductionnistes et positivistes, l'idée que l'homme est ancré dans la transcendance et qu'une négation de la transcendance dans sa vie l'a condamné à une vie de vide et de vanité absolue n'est pas prise en considération. De telles fausses anthropologies qui ne reconnaissent pas l'âme (en tant que fondement spirituel transcendant) à l'intérieur de l'homme, acceptant plutôt un modèle de "matrice matérielle » pour décrire l'être humain. Par conséquent, le soidisant « modèle spirituel » de l'homme, créé par Dieu à l'image de Dieu (imago Dei) et avec une âme infusée et un but conféré qui transcende ses besoins biologiques et psychologiques immédiats, est intentionnellement refusé. La matrice matérielle agit contre la communauté en soutenant un style de vie d'individualisme égoïste et affaiblit ainsi les relations entre les personnes. (Piwowarski, 1996)

La rupture des liens entre les personnes humaines est clairement démontrée par une rupture culturelle en cours : la dégradation des bonnes manières dans la vie politique, l'augmentation du nombre de personnes vivant seules, le faible taux de participation électorale, les niveaux élevés de criminalité et de violence, les crises familiales croissantes et tensions dans les relations interpersonnelles, etc. On attribue trop d'importance à la richesse économique, au pouvoir politique, au prestige social et aux plaisirs sensuels. Ces tendances se manifestent par le retrait de l'individu, avec ses buts malavisés et souvent matérialistes, d'une communauté humaine constitutive à son propre monde égoïste et surindividualiste. (Luzny, 2005) Il est important de noter que cela correspond à une compréhension relativiste de la vérité, à une approche

XLinguae, Volume 14 Issue 4, October 2021, ISSN 1337-8384, eISSN 2453-711X 
consumériste de l'amour (y compris un accent exagéré sur le corps physique humain), à l'instabilité des formes alternatives de vie collective, à la fuite du social responsabilité des politiciens et autres acteurs sociaux, la dégradation de la famille, l'augmentation du nombre de célibataires, la propagation de la méfiance parmi les citoyens, les niveaux élevés de criminalité, la violence croissante et la montée des cas de dépression. (Bogard, 2005; Lund - Dearing, 2013) Ces déterminants sont étroitement liés à une recherche incessante des bénéfices des individus, ce qui tend à contredire la plupart des efforts pour cultiver une existence communautaire authentique. (Nespor - Luzny, 2007)

Les vues anthropologiques dérivées de cette «matrice matérielle » ont non seulement remplacé une compréhension chrétienne de l'être humain, mais " elles représentent aussi... une tendance à créer des opinions convaincantes mais déformées sur qui nous sommes en choisissant un aspect de nous-mêmes et en le portant niveau de vérité supérieure concernant qui nous sommes et ce que nous faisons. La matrice matérielle introduite stimule l'individualisme égoïste et l'effondrement de la communauté » (Kuna-Fforde, 2006: xxiii). Cela entraine des sentiments aigus de solitude et d'isolement. (Fforde 2016). La désocialisation à grande échelle à laquelle nous assistons ne peut être contrée par la prescription de plus d'antidépresseurs, du moins pas à long terme et de manière durable. Le recours à des préoccupations plus intenses avec le corps humain ainsi que des expériences de plaisirs corporels intenses n'offriront pas non plus un antidote viable à la perte de sens qui découle de la déconnexion et de la fragmentation décrites ci-dessus.

La question qui semble commodément négligée est de savoir si ces phénomènes (c'est-à-dire les indicateurs socioculturels de la désocialisation) sont la conséquence ou la cause du déclin universel de la religiosité chrétienne dans notre domaine civilisationnel. On peut soutenir que l'entropie sociale en cours sous forme de désocialisation doit être traitée de manière holistique, c'est-à-dire en analysant l'utilisation (ou l'absence) des ressources religieuses pour l'épanouissement humain et social (cf.: Taylor 1989; 2007; Casanova, 2011). Nous soutenons en outre que la désocialisation est étroitement liée aux processus de sécularisation actuels, ainsi qu'à la progression d'une vision matérialiste de l'homme en conformité avec les modèles et les théories des anthropologies postmodernes.

La Slovaquie, avec d'autres pays d'Europe centrale et orientale, a été confrontée à l'idéologie matérialiste marxiste jusqu'en 1989. À partir des années 1990, le matérialisme de consommation (ou consumérisme matérialiste) est devenu le nouveau défi majeur. Cependant, ces deux perspectives idéologiques oppressives et réductionnistes ont une base commune - une interprétation réductionniste, biologicoreproductrice de l'être humain, qui implique un rejet de la transcendance. D'un point de vue idéologique, les sociétés post-communistes ne sont donc pas confrontées à un défi complètement nouveau car elles ont déjà connu un combat pour l'âme de l'homme sous le régime communiste. Cette expérience peut, dans certaines circonstances, constituer un avantage face au défi du matérialisme (occidental).

\section{La montée du culte du corps}

Bien que la tendance à souligner arbitrairement l'importance du corps dans un contexte désocialisé ait été observée et critiquée il y a des décennies, les médias de masse et les réseaux sociaux en ligne ont accéléré son développement et sa réception sociale au-delà des mesures au cours des 20 dernières années. Notre milieu socioculturel actuel nous donne l'impression que le soin du corps a été élevé au rang de culte d'adoration. Il semble que les humains contemporains ne voulaient pas accepter le vieillissement et la mort. Ce soin exagéré du corps montre des signes d'intérêt personnel narcissique. La devise d'aujourd'hui pourrait être formulée comme un désir prétendument auto-justifiable « d'être éternellement jeune et d'éviter de 
vieillir ", qui encourage l'individu à adopter des stratégies instrumentales pour lutter contre la détérioration et la décomposition [...] et la combine avec la notion que le corps est un véhicule de plaisir et d'expression de soi. Cela conduit alors à l'émergence d'une nouvelle norme sociale où les images omniprésentes du corps « belles, ouvertement sexuelles et associées à l'hédonisme, aux loisirs et à l'affichage, soulignent l'importance de l'apparence et du regard », en tant qu'éléments constitutifs indispensables de l'identité, statut et estime de soi. La culture contemporaine des médias de masse et des médias sociaux prospère en permettant et en favorisant directement de telles tendances, élevant ainsi de nouvelles générations d'individus superficiellement connectés dont la vie est presque irréversiblement liée à la réalité des médias. Une nouvelle culture de consommation imprégnée de médias émerge, où «les publicités, la presse populaire, la télévision et le cinéma, fournissent une prolifération d'images stylisées du corps. De plus, les médias populaires soulignent constamment les avantages de l'entretien du corps par les cosmétiques. La récompense pour le travail du corps ascétique cesse d'être le salut spirituel ou même une santé améliorée, mais devient une apparence améliorée et un moi plus commercialisable. (Featherstone, 1982: 18)

D'un point de vue spirituel, nous pourrions dire que la tension constructive entre le corps et l'esprit a pratiquement disparu. Le corps n'est plus restreint, mais plutôt « abondant » en tant que bien qui va de soi. Le corps devient à la fois sujet et objet. Ce processus de changement a incité les humains à essayer de prolonger la jeunesse et la vie par tous les moyens disponibles (Stegu, 2019; Nguyen et al., 2019; Pohar, 2019). D'un autre côté, alors qu'ils continuent de perdre leur soutien transcendantal ou religieux, les humains ne savent toujours pas comment faire face à leur propre limitation et mortalité (Klun, 2019; Osredkar, 2019). Une culture qui soutient cette tendance va à l'encontre des anciens paradigmes de ce que l'on peut appeler une « herméneutique de l'existence » ouverte à la transcendance qui cherche à " répondre à des questions de valeur et d'existence, concernant le sens de la vie, ou la recherche de formes expressives de l'homme. désir de la vie éternelle(Slivka, 2019: 174).

Au lieu d'un corps objectif, nous avons ici un corps psychologique. La conscience de soi de son propre corps est devenue un excellent exemple d'objectifs narcissiques, travaillant à faire exister le corps pour lui-même, à stimuler sa capacité à se refléter et à retrouver ce que nous pourrions appeler l'intériorité du corps ». D'où le désir de faire revivre toutes les manifestations possibles du corps humain, de l'aider à « communiquer » (pour ainsi dire), de découvrir son corps non seulement de l'extérieur mais aussi de l'intérieur, en cherchant la subjectivité du corps par différentes techniques, en cherchant le la beauté et le naturel du corps, etc. Les gens sont encouragés à ne ressentir ni honte ni réserves sur leur corps. Ces nouveaux accents aboutissent à l'émergence d'une nouvelle norme sociale selon laquelle il est à la fois normal et souhaitable d'exposer toutes les forces corporelles et de montrer toutes les parties de son corps. Paradoxalement, cette signification élevée de son corps est détachée de ses deux éléments constitutifs importants - la relationnalité et la reproduction. Cela se produit, par exemple, en " proposant que la sexualité humaine revienne aux formes les plus primitives de méthodes de reproduction asexuées et non relationnelles ». (Miller, 2001: 501) Ainsi, la solution par le Créateur à la solitude originelle d'Adam (en tant que représentation primordiale de l'humanité) sous forme de différenciation sexuelle qui constitue l'attraction mutuelle et « la fécondité au-delà d'une existence singulière et individuelle » (Miller, 2001: 505) est complètement niée.

\section{La destruction soigneusement orchestrée de la famille}

Au nom de la tolérance, l'intolérance la plus dure s'insinue tacitement dans le discours social actuel sous le couvert de la pluralité et de la liberté sifflées par les nouveaux idéologues. Cette idéologie politico-culturelle postmoderne est basée sur l'idée d'une

XLinguae, Volume 14 Issue 4, October 2021, ISSN 1337-8384, eISSN 2453-711X 
longue marche à travers [ou plutôt contre] les institutions établies, y compris la destruction de l'institution de la famille, la déchristianisation de la société (la désintégration de la morale fondée sur le judéo- Doctrine chrétienne de l'idéologie de la valeur), l'abolition de toute contrainte sexuelle et la destruction des traditions, du patriotisme et du capitalisme. Ces processus sont corrélés à la diffusion de la culture de la mort, qui cherche à corrompre, affaiblir et finalement détruire le bon fonctionnement de cette civilisation, car dans une communauté qui fonctionne mal, il est plus facile de semer et de multiplier ses idées hédonistes et égoïstes. Un tel objectif, cependant, est difficile à atteindre directement, c'est pourquoi une tâche plus élémentaire (et plus précisément ciblée) est choisie: la destruction de la pierre angulaire et du pilier de la société - la destruction de la famille.

La destruction de la famille est réalisée indirectement parce que la famille, en tant qu'institution socialement acceptée, a depuis longtemps une autorité ferme et inébranlable dans la culture européenne. La stratégie vise donc à détruire son pilier fondamental - un mariage stable. Le mariage stable est liquidé par une remise en question ou une dissolution graduelle de sa soi-disant colle biologique - la fidélité sexuelle. Une révolution sexuelle est invoquée et saluée comme une nouvelle libération. La révolution sexuelle, à travers la " dé-tabou » initiale du sexe et de la sexualité, conduit à une absolutisation toujours plus grande de la valeur de la sexualité, de l'expression sexuelle et du plaisir sexuel.

Dans l'esprit de cette stratégie peu profonde teintée d'idéologie, afin d'induire la révolution sexuelle et de maintenir ses résultats, les nouvelles élites culturelles doivent exercer le pouvoir des médias de masse et elles doivent mettre en place une éducation sexuelle appropriée (en manipulant de manière appropriée) dans les écoles, entre autres. Dans le même temps, ces manipulateurs doivent développer des technologies qui assurent une séparation fiable et complète de la sexualité de la reproduction (par les deux moyens, la contraception et l'avortement). Tout cela s'inscrit dans l'esprit des journaux orwelliens sous le concept de santé sexuelle et reproductive. Cela leur permet ensuite d'argumenter que la sexualité peut ne plus avoir de valeur instrumentale significative (en tant qu'outil, moyen de reproduction des personnes) car nous pouvons lui attribuer une valeur purement autonome (en tant que telle, elle peut être favorisée et louée pour elle-même, c'est-à-dire le plaisir qu'il procure aux gens). Enfin, lorsque l'on réfléchit à la menace potentielle que cette approche progressive constitue pour la reproduction humaine, les progrès actuels des technologies de la reproduction ne doivent pas être présentés au public comme une utopie, mais plutôt comme une "garantie de survie » potentielle. Puisqu'on peut s'attendre à ce que les les changements dans la perception des normes sociales liées à la sexualité et à la famille seront chargés de divers problèmes, une stratégie de mise en œuvre prudente doit être conçue: pour éviter une attention inutile, une évolution et une annonce systématiques de l'idéologie correspondante, et son institutionnalisation à travers des organisations telles que la Fédération internationale pour la planification familiale (IPPF), l'Organisation des Nations Unies (ONU), l'Organisation mondiale de la santé (OMS) ou le Parlement européen devront être promulgués.

\section{Lumières - Modernisme - Idéologie du scientisme}

Chaque fois que les humains essayaient de construire l'harmonie dans une société immorale en employant la soi-disant " raison nue » de la rationalité scientifique humaine, ils créaient quelque chose de si scandaleux, quelque chose de si aveugle et inhumain que toute la structure s'effondrait sous le fardeau de la malédiction humaine. La matrice matérialiste comprend les directions suivantes : humanisme, rationalisme, droitisme, sociétalisme, économisme, powerisme, animalisme, sexualisme, physiologisme, sentimentisme, psychisme, relativisme. L'Homo Sapiens est perçu comme un intellect ambulant et la raison humaine est censée être le seul intellect de l'univers. Il n'y a pas de mental supérieur au-dessus de l'homme. La foi et la sagesse 
traditionnelle sont moins valables et moins fiables. L'essence de ce processus est de lier fondamentalement le progrès avec la rationalité et la liberté et le processus de régression avec la religion. La consommation devient la valeur, le fondement et l'objectif principal de la vie d'un individu. L'individu est de plus en plus à la recherche de sens et d'épanouissement dans les valeurs matérielles et de consommation. On s'écarte de la réalisation des valeurs caractéristiques des sociétés traditionnelles: valeurs familiales (le mariage et la famille sont souvent remplacés par des soi-disant unions), la religion (les religions traditionnelles sont remplacées par une religiosité alternative, une nouvelle religiosité ou spiritualité), le patriotisme.

Dans le même temps, cependant, ce visage risqué évité de l'idéologie du modernisme de l'Europe occidentale (de la période des Lumières, en passant par l'explosion de la révolution sexuelle anarchiste, à la rhétorique postmoderne actuelle représentée par le néo-marxisme et l'école de Francfort) est un défi pour nous de reconsidérer la direction de l'espace interhumain et de la vie sociale de notre société ultramoderne. C'est aussi un défi pour la société slovaque, dont le gouvernement a fait des choix politiques en faveur de la civilisation de la mort. (Kardis, 2008)

La culture de la mort mène une guerre offensive, tandis que la culture de la vie mène une guerre défensive. Nous ne devons pas être confus lorsque les protagonistes de la culture de la mort cherchent à interpréter à tort chaque acte défensif de la culture de la vie comme un acte offensant. (Jean-Paul II, 2006) Ils le font par tous les moyens disponibles, y compris les mensonges et les tromperies. Nous avons systématiquement séparé la sexualité de la fertilité par la contraception et l'avortement. Lorsqu'ils sont confrontés aux conséquences désastreuses de cette approche apparemment progressiste, cependant, les liens entre le comportement sexuel et la crise démographique sont intentionnellement dissimulés. Ce tabou est protégé par la condamnation, la diffamation, la disqualification et les sanctions judiciaires. La contraception, l'avortement et les nouveaux agendas en matière de sexualité et de genre sont promus par les institutions les plus fortes du monde, notamment l'OMS, l'ONU et la Commission européenne. Le programme américain d'aide au développement réduit son aide au développement traditionnelle et investit à la place des milliards de dollars dans des programmes « reproductifs » pour la contraception (connue sous le nom de prévention), la stérilisation et l'avortement dans les pays pauvres. La sexualisation des jeunes commence dès la naissance avec le soutien des Nations Unies, dont l'UNICEF. L'homosexualisation de la population semble être à l'ordre du jour mondial de l'ONU et de l'UE. Une civilisation qui a longtemps eu la connaissance et le respect du bon sens, ferme maintenant son esprit aux signes des temps. Aucun don prophétique n'est nécessaire pour reconnaître la séquence causale et la cohérence logique de ce à quoi nous assistons:

1. La société sexuée a besoin de contraception et d'avortement.

2. La contraception systématique et l'impunité pour les massacres d'enfants à naître entraînent une réduction significative de la population.

3. La sexualisation de la population et l'abolition de toutes les normes sexuelles conduisent à la désintégration familiale.

4. La désintégration de la famille entraîne des sentiments massifs d'anxiété, de dépression et de troubles psychologiques et somatiques de toutes sortes, en particulier chez les jeunes.

Le chaos social qui résulte de la destruction des normes morales traditionnelles, de l'éthos et des institutions (principalement la famille) ne peut plus garantir le tissu social et moral nécessaire qui soutiendrait les communautés sociopolitiques humaines à long terme. Dans le pire des cas, une telle situation peut entraîner le type d'aliénation existentielle et de fragmentation sociopolitique propice à la montée d'une dictature.

XLinguae, Volume 14 Issue 4, October 2021, ISSN 1337-8384, eISSN 2453-711X 


\section{Contrer la culture de la mort}

L'idéologie décrite ci-dessus contredit donc la conception anthropologique fondamentale de l'humain en tant que personne dans son unité inséparable de corps, d'âme et d'esprit. La sexualité est un élément constitutif d'une personne. La masculinité ou la féminité est un cadeau que nous ne pouvons pas choisir, mais nous ne pouvons qu'accepter ou rejeter. Nous construisons notre identité sur la base de conditions génétiques et biologiques et la développons en tant que participants dans un environnement social (c'est-à-dire interpersonnel, relationnel) donné. Nous intégrons progressivement notre sexe et notre sexualité dans notre personnalité. Si nous ne l'acceptons pas, cela entraîne de graves dommages et déviations psychologiques, ce qui soulève certainement la nécessité de rechercher une théorie qui justifierait un tel échec. Le genre apparaît, entre autres, comme une aide pour justifier l'homosexualité. Contrairement à cela, le camp conservateur soutient qu'il n'y a qu'une seule sexualité, une qui implique une dimension de relation au sexe opposé, la soidisant altérité. Si cette altérité disparaît pour faire place à 1 '« homo » (similitude), la sexualité disparaît avec elle.

Dans son Evangelium Vitae (25 mars 1995), feu le pape Jean-Paul II (1995) soutenait de manière révélatrice que ceux qui pratiquent le culte moderne du corps légitiment des crimes abominables qu'aucune loi humaine ne peut autoriser: "Rien ni personne ne peut justifier le meurtre de un être humain innocent, qu'il s'agisse d'un fœtus ou d'un embryon, d'un enfant ou d'un adulte, âgé, incurablement malade ou mourant. [...] Aucune autorité ne peut légitimement l'imposer ou l'autoriser. » (Jean-Paul II, 1995: 57) L'avortement artificiel est - quelle que soit la manière dont il est pratiqué - «le meurtre conscient et direct d'un être humain à un stade précoce de sa vie entre la conception et la naissance. [...] Un être humain est assassiné au seuil de la vie, et donc la personne la plus innocente jamais imaginée : il ne peut donc jamais être considéré comme un agresseur, encore moins comme un envahisseur injuste ! » (JeanPaul II, 1995: 58).

Le fait que Dieu nous a créés à son image avec un corps nous invite à exprimer et à recevoir de l'amour à travers notre corps. Ceci est vrai pour toute personne créée et appelée par Dieu, mais est mieux représentée par la relation intime d'un couple marié. Les humains en tant qu'esprits incarnés reçoivent de l'amour et se donnent aux autres dans l'amour en créant des relations humaines concrètes et en vivant le mystère du mariage. Mais la raison la plus fondamentale pour laquelle la théologie considère le corps comme un sujet essentiel est le mystère de l'incarnation du Logos divin - le seul vrai sacrement et mystère suprême. A ce propos, le pape Jean-Paul II rappelle que : « Le sacrement, en tant que signe visible, se constitue avec l'homme, en tant que corps, au moyen de sa masculinité et de sa féminité visible. Le corps, et lui seul, est capable de rendre visible ce qui est invisible: le spirituel et le divin. Il a été créé pour transférer dans la réalité visible du monde le mystère caché depuis des temps immémoriaux en Dieu, et ainsi en être un signe. » (Jean-Paul II, 2006: 54)

Le point central de la théologie du corps du Pape peut être résumé de la manière suivante: l'homme et la femme existent en tant que dons l'un à l'autre dans et à travers le corps en tant que «nuptial » et sont donc à l'image et à la ressemblance de la Trinité, le divin communion des personnes... Ce don mutuel des personnes s'incarne parfois en une tierce personne, mais il doit toujours faire naitre une forme de vie nouvelle entre elles. Ce don doit être total, désintéressé et gratuit. Un tel don reflète le créateur. En tant que nuptial, le corps a la capacité d'exprimer l'amour: précisément cet amour dans lequel la personne humaine devient un don et, au moyen de ce don, remplit le sens même de son être et de son existence (Miller, 2001: 507). D'un point de vue théologique chrétien, le mariage n'est donc pas «l'effet du hasard ou le produit de l'évolution des forces naturelles inconscientes; c'est la sage institution du Créateur de réaliser dans l'humanité son dessein d'amour. Au moyen du don personnel réciproque 
de soi, qui leur est propre et exclusif, le mari et la femme tendent vers la communication de leurs êtres en vue d'une perfection personnelle mutuelle, à collaborer avec Dieu dans la génération et l'éducation de nouvelles vies » (Pape Paul VI, 1968: § 8)

\section{Vers la reconnaissance de notre personnalité incarnée}

La théologie mature du corps de Jean-Paul II découle de la conviction que la sensualité n'a pas besoin d'éloigner un être humain d'un autre. De même, il n'est pas nécessaire qu'elle constitue le moyen d'exploitation d'autrui pour le plaisir d'autrui. Comprendre cela nous fournira une base solide pour surmonter la peur de devenir des objets, que quelqu'un d'autre ne nous verra que comme des objets du désir humain. Le pape polonais révèle ici quelque chose de fondamental de l'être humain : nous, sujets personnels investis d'une dignité inaliénable, ne voulons pas être des objets humains. Nous voulons que les autres nous voient avec notre dignité et non comme de simples outils de satisfaction personnelle. (Jean-Paul II, 1993)

Il existe une dimension indélébile de transcendance de chaque personne humaine. Pas dans le sens de transcender la nature, ou de se transcender à travers mon unité avec la nature mais plutôt dans la transcendance d'une personne humaine à travers sa conscience. Nous servons la Vérité en étant ouverts à sa conscience. Cela va à l'encontre du matérialisme historique, qui croit que les conditions matérielles déterminent la condition de notre pensée. La corporalité est importante. Le corps humain est le moyen d'exprimer la personne. Mais notre vie n'est pas la vie des instincts. Nous devons clairement différencier les instincts et les pulsions. Il y a de l'émotivité légitime dans nos vies mais ce sont des dynamismes (au niveau de la sémantique et de la psyché) qui doivent être intégrés et soumis à de vraies valeurs, dictées par la conscience humaine. (Jean-Paul II, 2011)

La dimension corporelle peut nous servir de signe. Le corps participe à l'acte humain. Tous les dynamismes somatiques manifestent que l'humain appartient à ce monde visible. Le corps humain est l'expression du totus personae. « Le corps, en effet, et seul le corps est capable de rendre visible ce qui est invisible: le spirituel et le divin. Il a été créé pour transférer dans la réalité visible du monde le mystère caché de l'éternité en Dieu, et ainsi en être un signe » (Jean-Paul II, 2011: 19.4). Les personnes humaines ne peuvent pas exprimer leur sens et leur vocation sans le corps. Jean-Paul II nous invite en outre à entrer dans la dimension sacramentelle (signe visible d'une réalité invisible) en réfléchissant au mystère de notre incarnation. Le mystère de la vie de Dieu se manifeste lorsque le spirituel et le divin - un mystère caché de la perspective mondaine - est maintenant révélé dans l'existence incarnée de la personne agissante de Jésus de Nazareth et de tous ceux qui vivent comme des images vivantes de l'incarné. La dignité du corps et la valeur de toute la création sont ainsi établies. Toute création a une signification et une valeur plus profondes. Ce n'est pas seulement un moyen à notre disposition. De même, la parole du corps manifeste un sens. Le discours est lu principalement par la personne qui le prononce et ensuite aussi par ceux qui l'écoutent.

Jean-Paul II a critiqué à la fois les approches individualistes et anti-individualistes pour comprendre la nature de la personne humaine comme totalitaire. La seule solution viable qui émerge à la place est celle d'une " ontologie relationnelle », dans laquelle mon ultime acte personnel de liberté en tant que réalisation de moi-même de ma personnalité se produit lorsque je suis en relation avec les autres en respectant leur dignité et en répondant à leurs besoins. C'est une décision consciente d'être avec les autres, de vivre pour les autres, de se sacrifier pour les autres. Compte tenu de cette « perspective relationnelle ", notre définition de la vérité assume une fonction. La fonction de la vérité est de devenir une forme de vie des humains. La vérité est devenue chair. Pourquoi ? Pour que nous puissions le toucher, l'expérimenter,

XLinguae, Volume 14 Issue 4, October 2021, ISSN 1337-8384, eISSN 2453-711X 
l'engager existentiellement, humainement; afin que, dans une relation avec elle, nous puissions vivre dans la vérité, agissant comme des personnes humaines renouvelées. (Wojtyla, 1979) Notre cœur a été informé et formé par notre traitement raisonné d'expériences de vie significatives. La vie de vertu forme les habitudes du cœur, de l'esprit et du corps. Dans ce drame épique, l'activité humaine est ouverte à l'action de Dieu parce que nous sommes des êtres relationnels et poreux. (Valco et al., 2019; Valco, 2018) Nous ne manifestons pas seulement la vie et l'amour de la communion trine personarum; nous y participons! Ainsi, il devient évident que sans le Christ, le Logos primordial, il est impossible de reconnaître la vraie nature de la personne humaine.

\section{Conclusion}

Notre réflexion sur les implications de la modernité et de la postmodernité sur l'identité humaine vise à attirer notre attention sur la nécessité de définir un nouveau modèle d'intégrité sociale vers une éthos de dialogue et une identité socioculturelle basée sur la salubrité et l'équilibre du physique et métaphysique, sacré et aspects profanes, physiques et spirituels, rationnels et spirituels du micro et macro espace de la réalité sociale. Puisque nous avons affaire à des personnes, c'est-à-dire à des sujets moraux dans leur unicité et leur liberté, leur adhésion à la communauté communio (communauté humaine) doit être régie par la règle que l'anthropologie a formulée en tant que norme personnelle. Chaque individu humain est une personne et en tant que personne est unique, irremplaçable, irremplaçable et digne d'amour. La norme personnelle nous rappelle que la seule relation possible avec une personne en tant que personne est - d'aimer la personne. Seul ce type d'intégrité nous permet de maintenir la continuité de l'histoire et de construire une société démocratique stable, une communauté culturelle et spirituelle d'Europe.

La personne humaine porte une marque particulière du Créateur, étant créée à l'image même de Dieu comme une personne aimée et aimante, un être dans et pour les relations. Un signe peut indiquer un mystère; un sacrement, tel que le Logos incarné, réalise le mystère de Dieu. En tant que conjoints, nous nous aimons. Nous participons à la réalité de la fidélité et de l'amour de Dieu. C'est plus qu'une simple métaphore ou une signification. La possession de soi et l'auto-gouvernance appartiennent à la structure de la personnalité humaine. Mais cela ne veut pas dire que la personne est fermée, autoréférentielle, autonome. Il est plutôt ouvert à l'autre, n'émerge que par rapport à l'autre. Notre dépassement de soi par la participation crée alors une communauté de personnes. Il y a une participation à l'humanité des autres. Lorsqu'un «je» humain affirme le « je » de l'autre, « je le choisis en moi-même » (comme l'a soutenu Jean-Paul II). La personne humaine est ontologiquement ouverte à une autre personne. Il est essentiellement un " être poreux ». Nous existons en tant que personnes à cause de l'autre, avec l'autre et finalement pour l'autre. Nous acceptons l'autre dans notre vie même - le plus parfaitement en tant qu'époux. Nous devenons un seul corps avec le potentiel de générer une autre personne grâce à notre communion interpersonnelle d'amour.

\section{Acknowledgments}

This paper has been supported by the Kazan Federal University Strategic Academic Leadership Program.

\section{Bibliographic references}

BAK, T 2010. Mladez v kontexte novej nabozensko - moralnej skutocnosti, The youth in the context of new religious - moral reality. In: K. Kardis and M. Kardis (eds.). Nove nabozenske hnutia, sekty a alternativna spiritualita $\mathrm{v}$ kontexte postmoderny [New religious movements, sects and alternative spirituality in the context of postmodernity]. Presov: GTF PU, pp. 157-163. 
BAUMAN, Z. 2013. Liquid modernity. New Jersey: John Wiley \& Sons.

BAUMAN, Z. - HAUGAARD, M. 2008. Liquid modernity and power: A dialogue with Zygmunt Bauman. In: Journal of Power, vol. 1, n. 2, pp. 111-130.

BERGER, P. 2008. Religion and global civil society. In: J. Hanus, J. Vybiral (eds.). Nabozenstvi a globalni obcanska spolecnost. Brno: CDK, pp. 9-22.

BOGARD, K. L. 2005. Affluent adolescents, depression, and drug use: the role of adults in their lives. In: Family Therapy: The Journal of the California Graduate School of Family Psychology, vol. 32, n. 2, pp. 95-120.

CASANOVA, J., 2011. Public religions in the modern world. Chicago: University of Chicago Press.

FEATHERSTONE, M. 1982. The body in consumer culture. In: Theory, culture \& society, vol. 1, n. 2, pp. 18-33.

FFORDE, M. 2010. Desocializacia. Kriza postmodernity, Desocialization. A postmodern crisis. Bratislava: Luc.

FFORDE, M. 2016. Das Zeitalter der Einsamkeit: Entsozialisierung als Krise der modernen Gesellschaft. Freiburg im Breisgau: Verlag Herder GmbH.

GIDDENS, A. 2010. Dusledky modernity, The consequences of modernity. Praha: Slon.

JOHN PAUL II. 1993. Love and responsibility. San Francisco: Ignatius Press.

JOHN PAUL II. 1995. Evangelium Vitae [online]. Rome: Libreria Editrice Vaticana. Available online: http://www.vatican.va/content/john-paulii/en/encyclicals/documents/hf_jp-ii_enc_25031995_evangelium-vitae.html (cit. 20/12/2020).

JOHN PAUL II. 2006. The Redemption of the Body and Sacramentality of Marriage. Theology of the Body. (From the Weekly Audiences of His Holiness, September 5, 1979 - November 28, 1984). Washington DC: The Catholic Primer.

JOHN PAUL II. 2011. Man and Woman: He Created them. A Theology of the Body. M. Waldstein (transl.). Boston: Pauline Press.

KARDIS, M. 2008. Godnosc osoby ludzkiej jako podmiotu miedzynarodowych stosunkow gospodarczych [The dignity of human being as a subject of international economic relations]. In T. Guz, K.A. Klosinski, P. Marzec (eds.). Czlowiek, granice panstw, gospodarka. Man, borders of rule, economy. Lublin: Wydawnictwo KUL, pp. 45-58.

KLUN, B. 2019. Transhumanizem in transcendenca cloveka. In: Bogoslovni Vestnik, vol. 79, n. 3, pp. 589-600.

KUNA, M. - FFORDE, M. 2006. Slovensko, materializmus a desocializácia. Slovakia, Materialism and Desocialization. Ružomberok: The Catholic University in Ruzomberok.

LESTHAEGHE R. - SURKYN J. 2008. When history moves on: The foundations and diffusion of a second demographic transition. In: R. Jayakody, A. Thornton, and W. Axinn (eds.). International family change: Ideational perspectives. New York London: Lawrence Erlbaum Associates, pp. 81-118.

LUND, T. J. - DEARING, E. 2013. Is growing up affluent risky for adolescents or is the problem growing up in an affluent neighborhood?. In: Journal of Research on Adolescence, vol. 23, n. 2, pp. 274-282.

LUZNY, D. 2005. Individualizace a modernizace nabozenstvi, The Individualisation and modernisation of religion. In Moderne nabozenstvo [Modern religion]. Bratislava: Ustav pre vztahy statu a cirkví, pp. 239-253.

MILLER, P. J. 2001. The theology of the body: A new look at Humanae Vitae. In: Theology Today, vol. 57, n. 4, pp. 501-508.

MONGARDINI, C. 1992. The ideology of postmodernity. In: Theory, Culture \& Society, vol. 9, n. 2, pp. 55-65.

XLinguae, Volume 14 Issue 4, October 2021, ISSN 1337-8384, eISSN 2453-711X 
NESPOR, Z. - LUZNY, D. Sociologie nabozenstvi [Sociology of religions]. Praha: Portál.

NGUYEN, T. T. - TRUONG, Q. T. T. - VALCO, M. - KHVATOVA, M. A. TYAZHELNIKOV, A. A. 2020. Christian Theological Views on Industrial Revolutions and Related Ethical Challenges: A Western (And a Global) Perspective. In: Bogoslovni Vestnik, vol. 80, n. 1, pp. 177-188.

OSREDKAR, M. J. 2019. Religija kot izziv za transhumanizem. In: Bogoslovni vestnik, vol. 79, n. 3, pp. 657-668.

PIWOWARSKI, W. 1996. Socjologia religii, Sociology of religion. Lublin: RW KUL. POHAR, B. 2019. Transhumanizem v sluzbi clovekove odgovornosti do stvarstva. In: Bogoslovni vestnik, vol. 79, n. 3, pp. 643-656.

POPE PAUL VI. 1968. Humanae Vitae. Available online on NewAdvent.org at: https://www.newadvent.org/library/docs_pa06hv.htm (cit. 8/10/2020).

SLIVKA, D. 2019. Hermeneutics in the ancient Greek and Hellenistic culture. Ljubljana : KUD Apokalipsa.

STEGU, T. 2019. Transhumanizem in krscanska antropologija. In: Bogoslovni vestnik, vol. 79, n. 3. pp. 683-692.

TAYLOR, C. 1989. Sources of the Self: The Making of the Modern Identity. Cambridge, MA: Harvard University Press.

TAYLOR, C. 2007. A Secular Age. Cambridge Massachusetts - London, UK: Harvard University Press.

VALCO, M. 2018. Sekularizácia ako výzva pre tradičné náboženstvá Európy podl’a Charlesa Taylora, Secularization as a Challenge for Traditional Religions in Europe according to Charles Taylor. In: Historia Ecclesiastica. - ISSN 1338-4341, vol. 9, n. 1, pp. 173-190.

VALCO, M. - STURAK, P. - VALCOVA, K. - ZOZULAK, J. - DURA, I. 2019.

Socialny trinitarianizmus vo svetle Lutherovych dorazov v uceni o Trojici. In: Historia Ecclesiastica, vol. 10. n. 1, pp. 176-192.

WOJTYLA, Karol. 1979. The person: Subject and community. In: The Review of Metaphysics, vol. 33, n. 2, pp. 273-308.

ZAIDI, B. S. - MORGAN, P. 2017. The Second Demographic Transition Theory: A Review and Appraisal. In: Annual Review in Sociology, vol. 43, n. 2, pp. 473-492.

Published online 2017 May 19. doi: 10.1146/annurev-soc-060116-053442.

Words: 6573

Characters: 43071 (23,93 standard pages)

Prof. Dr. Kamil Kardis, PhD.

Department of Philosophy and Religious Studies

Greek Catholic Theological Faculty, University of Presov

Ul. Biskupa Gojdiča 2

08001 Presov

Slovak Republic

kamil.kardis@unipo.sk

Assoc. Prof. Dr. Maria Kardis, PhD.

Department of Historical Sciences

Greek Catholic Theological Faculty, University of Presov

Ul. Biskupa Gojdiča 2

08001 Presov

Slovak Republic

maria.kardis@unipo.sk 
Prof. Dr. Gabriel Pala, PhD.

Department of Applied Educology

Greek Catholic Theological Faculty, University of Presov

Ul. Biskupa Gojdiča 2

08001 Presov

Slovak Republic

gabriel.pala@unipo.sk

Prof. Dr. Tadeusz Bąk, PhD.

Faculty of Theology

Catholic University in Ruzomberok

Hlavna 89, 04121 Kosice

Slovak Republic

tedbak@wp.pl

Prof. PhDr. Michal Valco, PhD.

Department of Church History

Evangelical Lutheran Theological Faculty

Comenius University in Bratislava

Bartokova 8

81102 Bratislava

Slovak Republic

michal.valco@uniba.sk

Institute of Psychology and Education

Kazan Federal University

Kremlyovskaya Str. 18, RU - 420008

Kazan

Russian Federation 